\title{
Propuesta de medida del desempeño innovador: aplicación en las empresas innovadoras españolas
}

\section{A proposal for measuring the innovation performance: an application in spanish innovative firms}

\author{
Raquel Arévalo Tomé \\ BEgOÑa URGaL ${ }^{2}$ \\ MARÍA A. QuintÁs \\ Universidade de Vigo (España)
}

Recibido el 12 de noviembre de 2010, aceptado el 6 de octubre de 2011

$\mathrm{N}^{\mathrm{o}}$ de clasificación JEL: M15, O3

DOI: $10.5295 / \mathrm{cdg} .100267 \mathrm{ra}$

\section{Resumen:}

En este artículo se aporta una medida del grado de desempeño innovador de la empresa. Este indicador resume los efectos de las actividades de innovación tecnológica en los productos, en los procesos y en la sostenibilidad de la empresa. Los datos proceden de las empresas españolas, representadas en el Panel de Innovación Tecnológica (PITEC) del 2006, que han desarrollado actividades de innovación tecnológica en el período 20042006. Además, se realiza una caracterización de las empresas innovadoras españolas empleando el indicador propuesto.

Palabras clave:

Desempeño innovador, Índice de síntesis, Análisis de Correspondencias, Empresas innovadoras, Panel PITEC 2006.

Abstract:

This paper supplies a measure of the degree of firm's innovation performance. This indicator summarizes the impact of technological innovation activities on products, processes and sustainability of the firms. The data set used in this study was drawn up from the 2006 Panel on Technological Innovation (PITEC). The research focuses on firms that carried out a technological innovation from 2004 to 2006 . We also show a characterization of Spanish innovative firms using the proposed indicator.

Keywords:

Innovation performance, Synthesis index, Correspondence Analysis, Innovative firms, 2006 PITEC.

\footnotetext{
${ }^{1}$ Facultade de Económicas e Empresariais. Lagoas Marcosende s/n 36310 Vigo (Pontevedra). arevalo@uvigo.es; quintas@uvigo.es

${ }^{2}$ Escola de Enxeñería Industrial de Ingenieros Industriales. Lagoas Marcosende s/n 36310 Vigo (Pontevedra). burgal@uvigo.es
} 


\section{INTRODUCCIÓN}

La medida del desempeño de la innovación es una de las principales preocupaciones de la gestión empresarial actual, sin embargo, no todas las empresas disponen de un procedimiento sistemático o estándar que permita capturar los resultados de sus actividades de innovación tecnológica. De ahí que el desempeño de la innovación tienda a ser valorado ad hoc, existiendo una variación considerable en las medidas empleadas de un año a otro o de unos proyectos a otros.

En este artículo se crea un indicador para la medida del desempeño innovador que sintetiza los posibles efectos de las actividades de innovación tecnológica. Esta medida no sólo pretende mostrar los resultados de los esfuerzos pasados de la empresa en este ámbito, sino también constituir una orientación sobre lo que es necesario hacer para obtener resultados en el futuro. Es decir, con ella se trata de desentrañar el potencial de tales actividades para crear una ventaja competitiva sostenible.

La literatura académica aporta diferentes opciones para enfocar la medida del desempeño de la innovación, no existiendo un acuerdo acerca de la más adecuada. Así, hay autores como Griffin y Page $(1993,1996)$, Huang et al. (2004), Molina-Castillo y Munuera-Alemán (2009a, 2009b) y Blindenbach-Driessen et al. (2010) que se centran en la medida de los resultados obtenidos del desarrollo de nuevos productos. Otros, como Prajogo y Ahmed (2006), definen una medida del desempeño de la innovación de producto y de proceso. Y hay otros autores como Birchall y Tovstiga (2006) que consideran que una medida adecuada del desempeño de la innovación debe incluir además el impacto en la responsabilidad social de la empresa.

Nosotras consideramos que, dado el gran número de actividades y estrategias diferentes que comprende la innovación empresarial, una solución adecuada para medir su desempeño es la adoptada en el ámbito de la innovación a nivel país o región (Grupp y Mogee, 2004). Esto es, crear un indicador de síntesis que capture las distintas dimensiones que los autores han estimado relevantes para medir los resultados de la innovación.

En consecuencia, construimos un índice que sintetiza los posibles efectos obtenidos de las actividades de innovación tecnológica en los productos, en los procesos y en la responsabilidad social de la empresa.

Esta medida presenta una serie de ventajas. Al agregar los posibles efectos de las actividades de innovación tecnológica en distintos ámbitos de la empresa, aporta una imagen global de los resultados de tales actividades y facilita considerablemente su análisis. Así, por ejemplo, por un lado, permite comparar directamente el nivel de desempeño innovador alcanzado por distintos tipos de empresas y, por otro lado, posibilita estudiar la contribución o el impacto en el desempeño innovador de distintos factores determinantes.

Así pues, el objetivo principal de este trabajo es la creación de un indicador del desempeño innovador a nivel de empresa y el objetivo secundario es utilizar ese indicador para identificar las características de las empresas españolas más innovadoras. Para ello contamos con una muestra de 9.432 empresas españolas de distintos sectores, pertenecientes al Panel de Innovación Tecnológica (PITEC) del año 2006. Estas empresas han realizado alguna actividad de innovación tecnológica en el período 2004-2006. Entendiendo como innovación tecnológica la definición del Manual de Oslo (OCDE, 2005), es decir, la introducción de un nuevo, o significativamente mejorado, producto (bien o servicio) o proceso. 
Este artículo se estructura de la siguiente manera. A continuación, en la segunda sección, se revisa el planteamiento que se realiza en la literatura de la medida del desempeño innovador. En la tercera sección se explica la construcción e interpretación del indicador que proponemos para medir el desempeño innovador. En la cuarta sección se analiza, empleando nuestro indicador, el nivel de desempeño innovador alcanzado por las empresas españolas según sus características estructurales y sus políticas de I+D+i. Finalmente, en la quinta sección se presentan las principales conclusiones e implicaciones para los directivos de las empresas.

\section{EL DESEMPEÑO INNOVADOR: UNA REVISIÓN}

Tal y como ya hemos mencionado en la introducción, la literatura académica aporta diferentes opciones para enfocar la medida del desempeño de la innovación, no existiendo un indicador generalmente aceptado o un conjunto común de indicadores. En la Tabla 1 se incluyen algunas de las aportaciones existentes.

La mayoría de los autores se centran de manera específica en analizar el desempeño en el desarrollo de nuevos productos, observándose que habitualmente combinan medidas financieras (rentabilidad, contribución al beneficio), medidas de mercado (cuota de mercado, volumen de ventas, apertura de nuevos mercados y penetración en el mercado), y medidas relativas al producto (ampliación de gama, calidad y novedad) y al cliente (aceptación del cliente y satisfacción del cliente). Sin embargo, hay autores que definen una medida del desempeño de la innovación no sólo de producto sino también de proceso, por cuanto que productos y procesos están estrechamente vinculados (Prajogo y Ahmed, 2006). Utterback y Abernathy (1975) ponen de manifiesto que a menudo una innovación de producto lleva consigo una innovación de proceso o viceversa, aunque normalmente la innovación de producto tiene un mayor protagonismo al inicio del ciclo de vida del producto y la innovación de proceso en las etapas de madurez y declive.

Pero incluso hay autores que consideran que una medida del desempeño innovador debe abarcar otros aspectos a parte de los relacionados con los productos y los procesos. Así, Birchall y Tovstiga (2006) comparan y contrastan la visión de expertos académicos y de profesionales en gestión de la innovación sobre la manera de medir el desempeño innovador y una de sus conclusiones es que es importante considerar el impacto que tiene las actividades de innovación tecnológica en cuestiones relacionadas con la responsabilidad social de la empresa. Estas actividades están vinculadas al resultado social corporativo a través de distintos aspectos, como por ejemplo, la reducción de residuos y el uso de materiales menos contaminantes. Alegre et al. (2006) contemplan en su medida del desempeño de la innovación de producto el desarrollo de productos respetuosos con el medioambiente. Phillimore (2001) afirma que hay un vínculo necesario entre el aumento del resultado social corporativo y la incorporación de nuevas tecnologías. Jaffe y Palmer (1997) analizan el impacto de la regulación ambiental en las actividades innovadoras a nivel de industria, y encuentran una relación positiva entre los costes de cumplimiento de la normativa y los gastos de I+D. No obstante, aunque la relación entre la estrategia corporativa y los aspectos sociales ha sido estudiada por los investigadores, el papel de la innovación en esta relación ha recibido poca atención (Pavelin y Porter, 2008). 
Un análisis detenido de las aportaciones incluidas en la Tabla 1 permite observar un cierto acuerdo entre los autores en cuanto a la manera de medir el desempeño de la innovación.

Así, la mayor parte de los autores están de acuerdo en la naturaleza multidimensional del desempeño innovador y en el uso de escalas multi-ítem para definir las distintas dimensiones. Las dimensiones consideradas por los autores varían, fundamentalmente, dependiendo del nivel de análisis: empresa, programa o proyecto. Por ejemplo, Hooley et al. (2005) y Chen et al. (2006) miden el desempeño a nivel de empresa, De Brentani y Kleinschmidt (2004), Atuahene-Gima et al. (2006) y Lichtenthaler (2009) lo hacen a nivel de programa, y Kusunoki et al. (1998), Lee y O’Connor (2003) y Blindenbach-Driessen et al. (2010) a nivel de proyecto.

Otro aspecto en el que parecen estar de acuerdo la mayoría de los autores es en el empleo de indicadores subjetivos del desempeño, esto es, basados en las valoraciones de los directivos (director de producto o de proyecto, director de I+D o director general). Sin embargo, hay autores como Ahuja y Katila (2001), Souitaris (2002), Hagedoorn y Clood (2003) y Chen et al. (2011) que optan por indicadores objetivos del desempeño, del tipo de: número de patentes registradas, o número de nuevos productos y procesos introducidos, en un determinado período de tiempo. Aunque el uso de indicadores subjetivos tiene inconvenientes bien conocidos, también presenta ventajas. Éstos permiten evaluar el desempeño de los procesos de innovación que todavía están en curso, puesto que se basan en valorar el logro de objetivos intermedios y/o resultados finales esperados. Hay que tener en cuenta que ciertas empresas, debido a que son muy jóvenes o a que el tiempo de desarrollo del producto es largo, no disponen de productos en el mercado, tan solo productos en desarrollo. Por lo que respecta en particular a uno de los indicadores objetivos más empleados, el número de patentes registradas, éste presenta limitaciones como medida del desempeño. Las empresas no siempre patentan sus invenciones, y las que son patentadas difieren en valor económico. Asimismo, hay invenciones que no son patentables.

Asimismo, por lo general, los autores al construir la medida del desempeño de la innovación otorgan la misma importancia a cada indicador. Sin embargo, Molina-Castillo y Munuera-Alemán (2009a) apuntan que los directivos no atribuyen la misma importancia a los diferentes indicadores de desempeño y que esta importancia puede variar dependiendo del horizonte temporal considerado. Este aspecto sí fue tenido en cuenta por Lee y O'Connor (2003). Estos autores consideran que la importancia o prioridad de los indicadores de desempeño varía en función del ciclo de vida del producto, de modo que cada indicador es evaluado en el corto plazo (en la fase de introducción y crecimiento del ciclo de vida del producto, cuando el volumen de ventas está creciendo) y en el largo plazo (una vez el producto ha alcanzado la fase de madurez y las ventas comienzan a estancarse).

También existe un cierto acuerdo entre los autores en cuanto al criterio adoptado a la hora de medir el desempeño de la innovación: eficacia o eficiencia. La mayoría de los autores opta por medidas de eficacia, al interesarse por los resultados que se obtienen de los procesos de innovación. Sin embargo, hay autores como Kusunoki et al. (1998), De Brentani y Kleinschmidt (2004), Alegre et al. (2006), Prajogo y Ahmed (2006) y Chen et al. (2011) que incluyen, en su medida del desempeño de la innovación, indicadores de eficiencia, relativos a los recursos consumidos para la obtención de unos resultados, como 
el coste y tiempo de desarrollo de productos, es decir, miden la productividad del proceso de desarrollo.

Nosotras compartimos los argumentos planteados sobre la importancia de considerar aspectos relativos a la sostenibilidad de la empresa en la medida de sus resultados de innovación. Además, estamos de acuerdo en la necesidad de incluir los efectos sobre los productos y los procesos de la empresa. Por todo ello, en este trabajo proponemos un indicador del desempeño innovador que integra los efectos que la innovación tecnológica provoca en los productos, en los procesos y en la responsabilidad social de la empresa. Por otra parte, optamos por una medida de desempeño innovador centrada en la eficacia, por cuanto que se refiere a la consecución de determinados resultados de las actividades de innovación. Hemos preferido incluir medidas de eficacia por cuanto que contemplamos el indicador propuesto como un instrumento de control en la gestión de las actividades de innovación tecnológica, que permita evaluar si la empresa ha logrado los objetivos que se ha fijado en este ámbito.

Asimismo, para clarificar el nivel del análisis realizado, siguiendo a García y Calantone (2002), optamos por una perspectiva micro al considerar como nuevos productos aquellos que fueron novedad para la empresa o para el mercado en el período considerado. Por otra parte, adoptamos una perspectiva de marketing y tecnológica, puesto que la innovación puede suponer un nuevo enfoque de marketing (nuevos mercados y/o nuevas habilidades y recursos de marketing) y/o un nuevo enfoque tecnológico (nuevos recursos de I+D y/o nuevos procesos de producción). ${ }^{1}$

\footnotetext{
${ }^{1}$ En García y Calantone (2002) se pueden encontrar diversos niveles de análisis clarificando los mismos y las implicaciones en cuanto al grado de innovación y los resultados empresariales.
} 


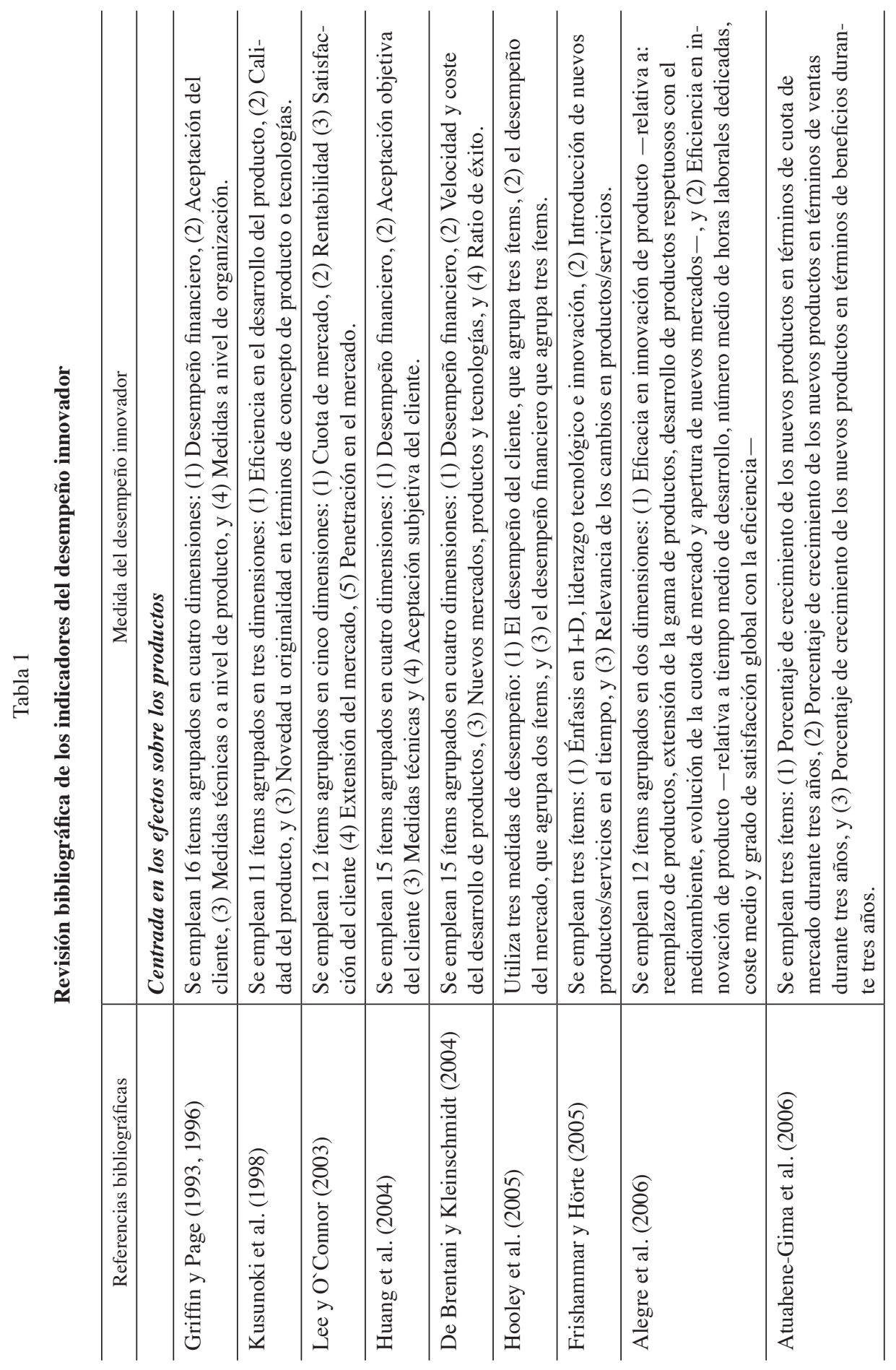




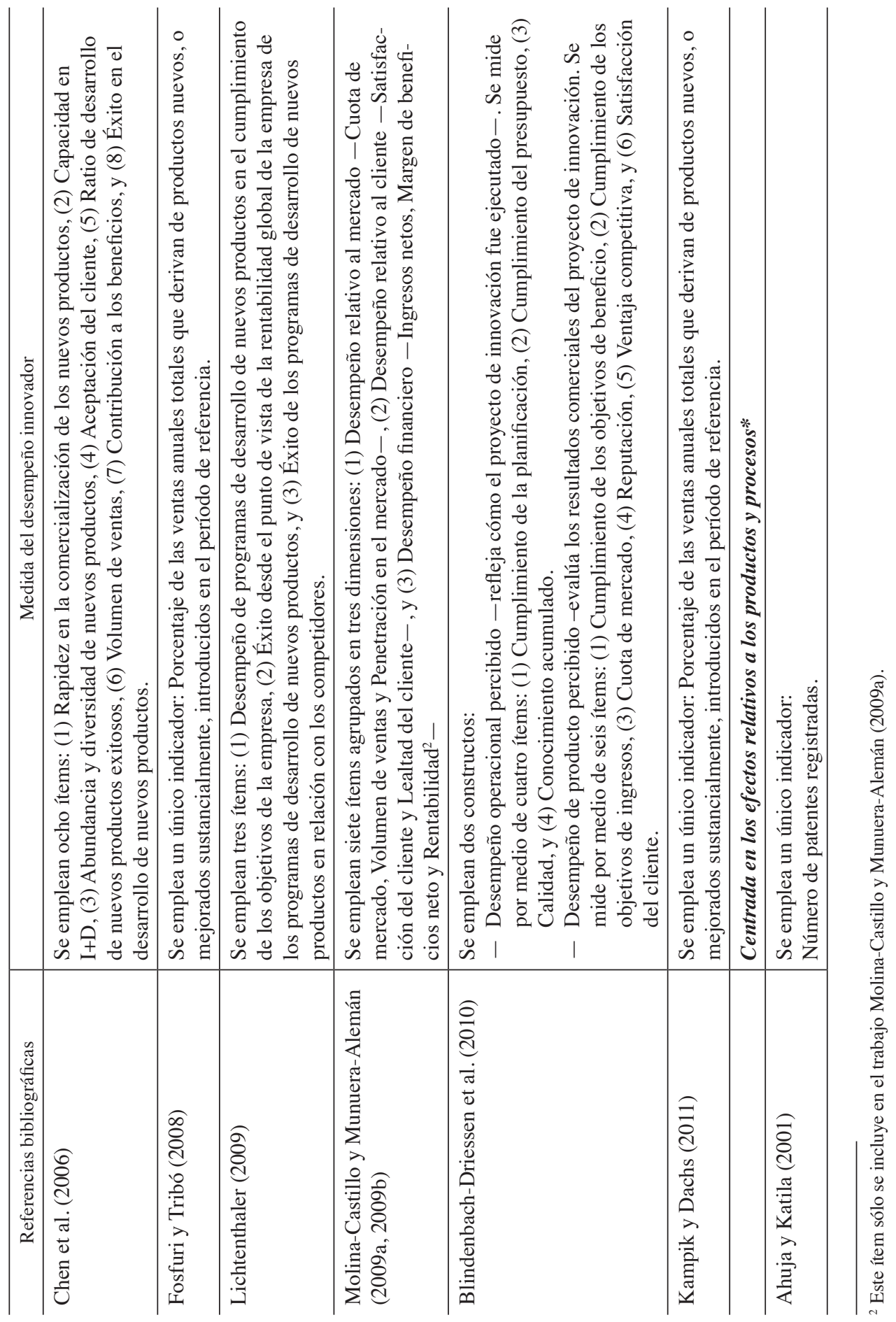




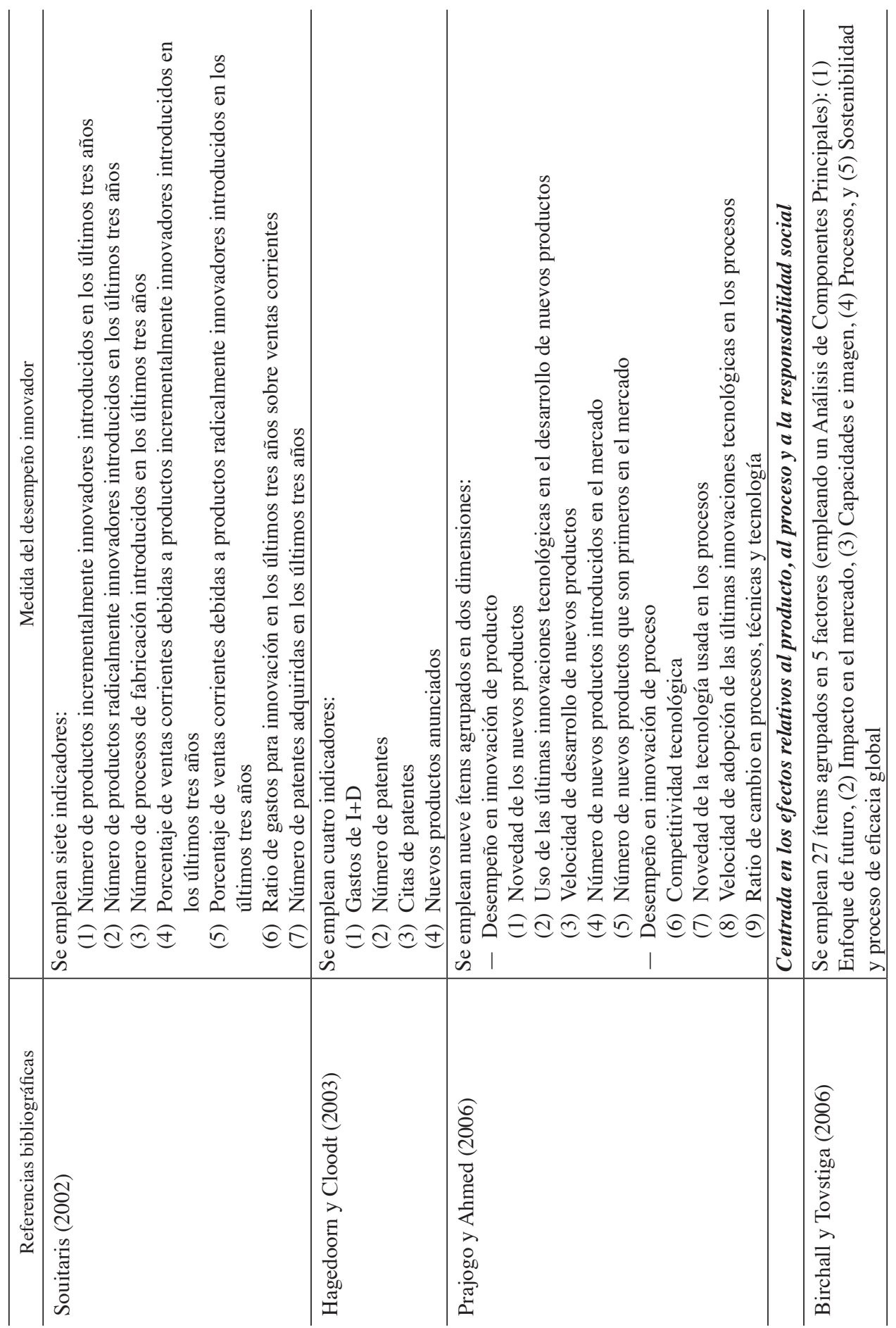




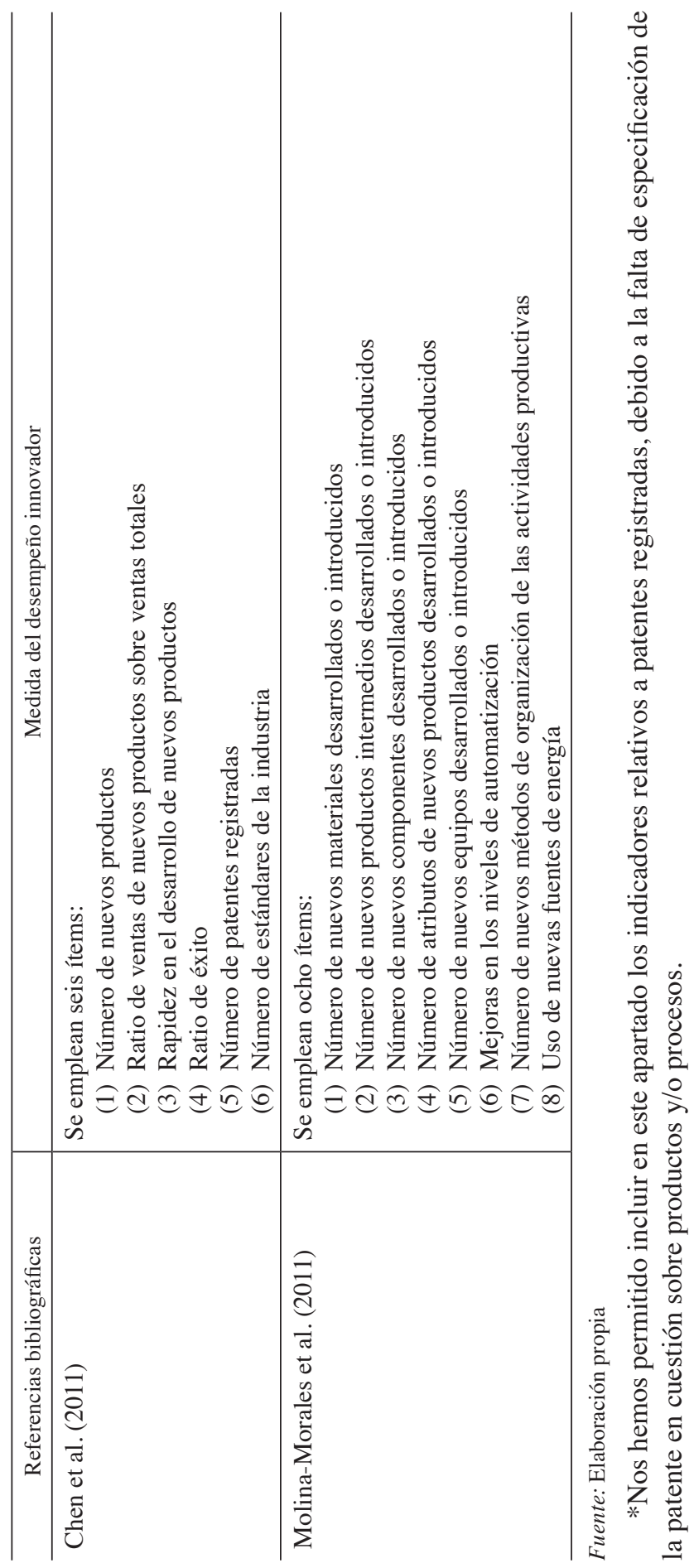




\section{CONSTRUCCION DE UN ÍNDICE DEL DESEMPEÑO INNOVADOR}

\subsection{Fuente de datos}

Los datos utilizados en esta investigación proceden del Panel de Innovación Tecnológica (PITEC) de 2006. El PITEC se enmarca dentro del Plan General de Estadísticas de Ciencia y Tecnología propugnado por la oficina de Estadísticas de la Unión Europea (Eurostat).

En lo que respecta a España, el PITEC constituye un instrumento estadístico sobre el seguimiento de las actividades de innovación tecnológica en nuestro país fruto del esfuerzo conjunto de tres instituciones: el Instituto Nacional de Estadística (INE), la Fundación Española para la Ciencia y la Tecnología (FECYT) y la Fundación Cotec. El objetivo de este proyecto es contribuir a mejorar la información estadística disponible sobre la actividad tecnológica de las empresas y las condiciones para la realización de investigaciones científicas en esta área.

Nuestro interés en este estudio se centra en aquellas empresas que han realizado actividades de innovación tecnológica en el período 2004-2006, cuyo número se eleva a un total de 9.432 empresas, representando un $73,68 \%$ del total de las empresas que componen el PITEC de 2006. En la Tabla 2 se muestran sus principales características descriptivas. Además de la distribución sectorial desagregada en 20 sectores, podemos observar que en el conjunto de empresas con actividad innovadora en España predomina la pequeña empresa (prácticamente el 53\% cuenta con 50 empleados o menos), la titularidad privada (que representa el $96 \%$ del total) y el ámbito de actuación internacional (que está presente en prácticamente el $63 \%$ de la muestra analizada).

Tabla 2

Descripción de la muestra (9.432 empresas con actividades en innovación tecnológica)

$\%$ sobre el total

Tamaño de la empresa:

$\begin{array}{rr}\text { Hasta } 10 \text { trabajadores } & 13,79 \\ \text { Entre } 11 \text { y } 50 & 39,09 \\ \text { Entre } 51 \text { y } 100 & 14,76 \\ \text { Entre 101 y 200 } & 10,75 \\ \text { Más de 200 } & 21,61\end{array}$

Tipo de empresas:

$\begin{array}{rr}\text { Pública } & 2,23 \\ \text { Privada nacional } & 86,06 \\ \text { Privada con participación extranjera } & 10,17 \\ \text { Asociación } & 1,55\end{array}$


$\%$ sobre el total

Sectores de actividad (Identificación CNAE):

$$
\begin{array}{rr}
\text { Industrias extractivas y del petróleo }(10,11,12,13,14,23) & 0,50 \\
\text { Alimentación, bebidas y tabaco (15, 16) } & 7,04 \\
\text { Textil, confección, cuero y calzado (17 a 19) } & 3,74 \\
\text { Madera, papel, edición y artes gráficas (20 a 22) } & 3,26 \\
\text { Química (24) } & 7,99 \\
\text { Caucho y materias plásticas (25) } & 3,73 \\
\text { Productos minerales no metálicos diversos (26) } & 3,23
\end{array}
$$$$
\text { Metalurgia (27) } \quad 1,60
$$$$
\text { Manufacturas metálicas (28) } \quad 5,89
$$$$
\text { Fabricación de maquinaria y material de transporte (29 a 35) } \quad 19,16
$$$$
\text { Manufacturas diversas (36) } \quad 2,79
$$$$
\text { Reciclaje (37) } \quad 0,37
$$$$
\text { Energía y agua }(40,41) \quad 0,80
$$$$
\text { Construcción (45) } \quad 3,03
$$$$
\text { Comercio y hostelería }(50,51,52,55) \quad 6,26
$$$$
\text { Transporte y almacenamiento (60 a 63) } \quad 1,51
$$$$
\text { Comunicaciones (64) } \quad 0,66
$$$$
\text { Intermediación financiera (65 a 67) } \quad 1,98
$$$$
\text { Actividades inmobiliarias, servicios a empresas (70 a 74) } \quad 21,64
$$

Servicios públicos, sociales y colectivos (80 - excepto 8030 - , 85,90 a 93) $\quad$ 4,84

Ámbito de actuación de la empresa:

$$
\begin{array}{rc}
\text { Local o autonómico } & 9,21 \\
\text { Nacional } & 27,86 \\
\text { Internacional } & 62,93
\end{array}
$$

Fuente: Elaboración propia a partir del Panel de Innovación Tecnológica (PITEC) de 2006

\subsection{Metodología}

Aplicando la técnica de Análisis de Correspondencias (AC) construimos un indicador del desempeño innovador. El AC es una técnica exploratoria válida para sintetizar la información relevante disponible en un conjunto de variables de carácter cualitativo. Proporcio- 
na indicadores obtenidos de la combinación lineal de las variables originales ${ }^{3}$ y resulta interesante cuando se dispone de un gran número de variables: (1) que presentan una elevada correlación entre sí y, (2) que son susceptibles de ser resumidas por una, o pocas variables que recojan la misma información. Por tanto, este análisis puede ser útil cuando el conjunto de variables: (1) hace referencia a diversas características de un determinado elemento o, (2) aporta datos con cierto grado de complementariedad sobre un mismo fenómeno.

Las variables utilizadas en este análisis recogen la valoración que declaran las 9.432 empresas (según el PITEC del 2006) que componen la muestra sobre los efectos obtenidos de sus actividades de innovación tecnológica en el periodo 2004-2006. El tipo de efectos sometidos a valoración hacen referencia a distintos aspectos relativos a los productos, a los procesos de producción y a la responsabilidad social de la empresa. En la Tabla 3 se describen las variables de nuestro estudio y en la Tabla 4 se muestran los principales estadísticos descriptivos que permiten justificar la idoneidad de la técnica aplicada. Así, en primer lugar se puede comprobar que en todos los casos el coeficiente de correlación es distinto de cero para un nivel de significación del 1\%, lo que da sentido a plantearse la construcción de un indicador que sintetice dichas correlaciones. También en el análisis previo sobre la validez y fiabilidad de los ítem utilizados se obtuvo una elevada covarianza media inter-ítem (cuyo valor es 0,48 ) y un elevado coeficiente alpha de Cronbach (cuyo valor es 0,86 ), mostrando una adecuada validez de la técnica aplicada a los datos disponibles.

Los resultados del AC se presentan en la Tabla 5 una vez se han llevado a cabo las correcciones propuestas en Greenacre (1991) con objeto de interpretar correctamente los resultados obtenidos directamente del AC cuando el número de variables es superior a dos. En la tabla se incluye la notación y la frecuencia de las respuestas según la valoración obtenida (columnas 2 y 3 , respectivamente) y los resultados del AC en relación al primer

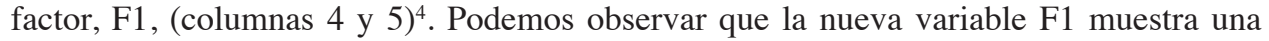
capacidad de síntesis muy relevante puesto que ella sola captura el $74 \%$ de la inercia o varianza total a explicar. En cuanto a su interpretación, en primer lugar, se observa que presenta una alta correlación con las nueve variables que intervienen en el análisis. Por tanto, todas las variables resultan importantes a la hora de ayudar a determinar el significado de $\mathrm{F} 1$, especialmente las relacionadas con los efectos derivados de la eficiencia de los procesos en la utilización de recursos, Menores costes laborales y Menos recursos, para las que la correlación con F1 es de 0,49 y 0,48, respectivamente. En segundo lugar, al analizar el peso de las cuatro posibles valoraciones de cada efecto se observa que se cumple un mismo patrón en todos ellos: (1) cuando la empresa adjudica un valor nulo o no relevante al efecto en cuestión, el peso es negativo y (2) cuando la empresa expresa un incremento del valor obtenido también se ve incrementado el peso adjudicado al F1. Nos encontramos, por tanto, con un factor que presenta un mayor valor en la medida en que la empresa valora más positivamente el efecto obtenido en cada uno de los aspectos evaluados.

\footnotetext{
${ }^{3}$ El AC es similar al Análisis de Componentes Principales (ACP), la diferencia está en el tipo de variables que determina su correcta aplicación. En el AC las variables deben ser de tipo cualitativo o discreto, mientras que en el ACP deben ser de tipo cuantitativo o continuo, además de seguir una distribución normal. La principal ventaja del AC frente al ACP, además de que no se requiere ningún supuesto sobre la distribución de las variables, es que cualquier variable cuantitativa puede ser transformada en cualitativa, al revés no.

${ }^{4}$ Restringimos la presentación de resultados al primer factor por ser el único relevante en términos interpretativos, no obstante, cualquier lector puede solicitar los resultados completos del AC a la dirección de correo-e: arevalo@uvigo.es.
} 
Tabla 3

Descripción de variables

\begin{tabular}{|c|c|c|}
\hline Variable & Notación & Valor \\
\hline \multicolumn{2}{|l|}{ Efectos sobre los productos: } & \multirow{6}{*}{$\begin{array}{l}\text { 0 si el efecto } \\
\text { no se produce o } \\
\text { es considerado } \\
\text { como irrele- } \\
\text { vante para la } \\
\text { empresa; }\end{array}$} \\
\hline $\begin{array}{l}\text { Ampliación de la gama de bienes } \\
\text { o servicios }\end{array}$ & Ampliación de la gama & \\
\hline $\begin{array}{l}\text { Penetración en nuevos mercados } \\
\text { o mayor cuota de mercado }\end{array}$ & Ampliación de mercados & \\
\hline $\begin{array}{l}\text { Mayor calidad de los bienes o } \\
\text { servicios }\end{array}$ & Mejora de calidad & \\
\hline \multicolumn{2}{|l|}{ Efectos sobre los procesos: } & \\
\hline $\begin{array}{l}\text { Mayor flexibilidad en la produc- } \\
\text { ción o la prestación de servicios }\end{array}$ & Mayor flexibilidad & \\
\hline $\begin{array}{l}\text { Mayor capacidad de producción } \\
\text { o prestación de servicios }\end{array}$ & Mayor capacidad & \multirow{3}{*}{$\begin{array}{l}\mathbf{1} \text { si el efecto } \\
\text { es considerado } \\
\text { como reducido; } \\
\mathbf{2} \text { si el efecto es } \\
\text { intermedio, }\end{array}$} \\
\hline $\begin{array}{l}\text { Menores costes laborales por } \\
\text { unidad producida }\end{array}$ & Menores costes laborales & \\
\hline $\begin{array}{l}\text { Menos materiales y energía por } \\
\text { unidad producida }\end{array}$ & Menos recursos & \\
\hline \multicolumn{2}{|l|}{ Efectos sobre la responsabilidad social: } & \multirow{3}{*}{$\begin{array}{l}3 \text { si el efecto es } \\
\text { elevado }\end{array}$} \\
\hline $\begin{array}{l}\text { Menor impacto medioambien- } \\
\text { tal o mejora en la salud y la } \\
\text { seguridad }\end{array}$ & Alcance de mejoras & \\
\hline $\begin{array}{l}\text { Cumplimiento de los requisitos } \\
\text { normativos }\end{array}$ & Cumplimiento normativo & \\
\hline
\end{tabular}

Fuente: Elaboración propia a partir del PITEC 2006.

Tabla 4

Estadísticos descriptivos de las variables

\begin{tabular}{|c|c|c|c|c|c|c|c|c|c|c|c|c|c|}
\hline \multirow[b]{2}{*}{ Variables } & \multirow[b]{2}{*}{ Min. } & \multirow[b]{2}{*}{ Max. } & \multirow[b]{2}{*}{ Media } & \multicolumn{2}{|c|}{ Desviación } & \multicolumn{7}{|c|}{ Correlaciones (* Nivel de significación al 1\%) } & \multirow[b]{2}{*}{ (9) } \\
\hline & & & & típica & (1) & (2) & (3) & (4) & (5) & (6) & (7) & (8) & \\
\hline Ampliación de la gama (1) & 0 & 3 & 1,83 & 1,11 & 1 & & & & & & & & \\
\hline Ampliación de mercados (2) & 0 & 3 & 1,62 & 1,12 & $0,67 *$ & 1 & & & & & & & \\
\hline Mejora de calidad (3) & 0 & 3 & 1,99 & 1,07 & $0,50 *$ & $0,54 *$ & 1 & & & & & & \\
\hline Mayor flexibilidad (4) & 0 & 3 & 1,60 & 1,04 & $0,23 *$ & $0,26^{*}$ & $0,37 *$ & 1 & & & & & \\
\hline Mayor capacidad (5) & 0 & 3 & 1,63 & 1,08 & $0,24 *$ & $0,30 *$ & $0,38^{*}$ & $0,66^{*}$ & 1 & & & & \\
\hline Menores costes laborales (6) & 0 & 3 & 1,22 & 1,04 & $0,25^{*}$ & $0,32 *$ & $0,36^{*}$ & $0,55^{*}$ & $0,61 *$ & 1 & & & \\
\hline Menores recursos (7) & 0 & 3 & 1,03 & 1,00 & $0,25^{*}$ & $0,31 *$ & $0,35^{*}$ & $0,45^{*}$ & $0,49^{*}$ & $0,69 *$ & 1 & & \\
\hline
\end{tabular}




\begin{tabular}{|c|c|c|c|c|c|c|c|c|c|c|c|c|}
\hline \multirow[b]{2}{*}{ Variables } & \multirow[b]{2}{*}{ Min. } & \multirow[b]{2}{*}{ Max. } & \multirow[b]{2}{*}{ Media } & \multicolumn{2}{|c|}{ Desviación } & \multicolumn{7}{|c|}{ Correlaciones (* Nivel de significación al 1\%) } \\
\hline & & & & típica & (1) & (3) & (4) & (5) & (6) & (7) & (8) & (9) \\
\hline Alcance de mejoras (8) & 0 & 3 & 2,97 & 1,14 & $0,26^{*}$ & $0,31 * 0,35^{*}$ & $0,31 *$ & $0,36^{*}$ & $0,42 *$ & $0,54 *$ & 1 & \\
\hline Cumplimiento normativo (9) & 0 & 3 & 1,27 & 1,20 & $0,30 *$ & $0,34 * 0,39 *$ & $0,32 *$ & $0,35^{*}$ & $0,41 *$ & $0,45^{*}$ & $0,67 *$ & 1 \\
\hline \multicolumn{4}{|l|}{ Covarianza media inter-ítem } & & 0,48 & & & & & & & \\
\hline \multicolumn{4}{|l|}{ Coeficiente alpha de Cronbach } & & 0,86 & & & & & & & \\
\hline
\end{tabular}

Fuente: Elaboración propia a partir del PITEC 2006.

Con estos resultados podemos interpretar F1 como un indicador global de los efectos de la innovación tecnológica para la empresa ya que, además de representar una notable capacidad de síntesis, existe una coherencia sistemática, para todos los efectos considerados, entre la valoración de las empresas y la ponderación en el F1. A partir de ahora nos referiremos a F1 como nuestro indicador de desempeño innovador.

Tabla 5

Aplicación del Análisis de Correspondencias (AC) sobre los efectos de la innovación tecnológica

\begin{tabular}{|c|c|c|c|c|}
\hline \multirow{2}{*}{$\begin{array}{c}\text { Variables originales } \\
(\text { Aspectos valorados del } 0 \text { al 3)* }\end{array}$} & \multirow[b]{2}{*}{$\begin{array}{l}\text { Notación según } \\
\text { valoración }\end{array}$} & \multirow[b]{2}{*}{$\begin{array}{c}\text { Frecuencia } \\
\text { observada }\end{array}$} & \multicolumn{2}{|c|}{ Primer Factor $(\mathrm{F} 1) * *$} \\
\hline & & & Pesos en F1 & $\begin{array}{l}\text { Correlación } \\
\text { (F1,variable) }\end{array}$ \\
\hline \multicolumn{5}{|l|}{ Efectos sobre los productos: } \\
\hline \multirow{5}{*}{ Ampliación de la gama } & P_gama-0 & 18,18 & $-3,25$ & \multirow{4}{*}{0,36} \\
\hline & P_gama-1 & 17,51 & 1,07 & \\
\hline & P_gama-2 & 27,5 & 6,23 & \\
\hline & P_gama-3 & 36,81 & 7,30 & \\
\hline & P_mercado-0 & 23,21 & $-4,26$ & \multirow{4}{*}{0,42} \\
\hline \multirow{3}{*}{ Ampliación de mercados } & P_mercado-1 & 18,85 & 4,18 & \\
\hline & P_mercado-2 & 30,71 & 6,74 & \\
\hline & P_mercado-3 & 27,23 & 7,83 & \\
\hline \multirow{4}{*}{ Mejora de calidad } & P_calidad-0 & 15,36 & $-6,00$ & \multirow{4}{*}{0,42} \\
\hline & P_calidad-1 & 12,35 & 0,97 & \\
\hline & P_calidad-2 & 30,7 & 5,52 & \\
\hline & P_calidad-3 & 41,59 & 7,47 & \\
\hline \multicolumn{5}{|l|}{ Efectos sobre los procesos: } \\
\hline \multirow[t]{4}{*}{ Mayor flexibilidad } & PC_flexibilidad-0 & 18,67 & $-4,49$ & \multirow{4}{*}{0,41} \\
\hline & PC_flexibilidad-1 & 25,51 & 2,28 & \\
\hline & PC_flexibilidad-2 & 32,58 & 7,31 & \\
\hline & PC_flexibilidad-3 & 23,24 & 8,06 & \\
\hline
\end{tabular}




\begin{tabular}{|c|c|c|c|c|}
\hline \multirow{2}{*}{$\begin{array}{c}\text { Variables originales } \\
\text { (Aspectos valorados del } 0 \text { al } 3 \text { )* }\end{array}$} & \multirow{2}{*}{$\begin{array}{l}\text { Notación según } \\
\text { valoración }\end{array}$} & \multirow{2}{*}{$\begin{array}{c}\text { Frecuencia } \\
\text { observada }\end{array}$} & \multicolumn{2}{|c|}{ Primer Factor $(\mathrm{F} 1) * *$} \\
\hline & & & Pesos en F1 & $\begin{array}{l}\text { Correlación } \\
\text { (F1,variable) }\end{array}$ \\
\hline \multirow{8}{*}{ Menores costes laborales } & PC_capacidad-0 & 21,02 & $-5,31$ & \multirow{4}{*}{0,45} \\
\hline & PC_capacidad-1 & 20,82 & 3,50 & \\
\hline & PC_capacidad-1 & 32,08 & 7,15 & \\
\hline & PC_capacidad-2 & 26,08 & 8,02 & \\
\hline & PC_trabajo-0 & 31,64 & $-3,93$ & \\
\hline & PC_trabajo-1 & 28,24 & 5,93 & \\
\hline & PC_trabajo-2 & 26,66 & 8,68 & 0,49 \\
\hline & PC_trabajo-3 & 13,46 & 9,30 & \\
\hline \multirow[t]{4}{*}{ Menos recursos } & PC_recursos-0 & 38,7 & $-2,71$ & \multirow{4}{*}{0,48} \\
\hline & PC_recursos-1 & 29,19 & 6,74 & \\
\hline & PC_recursos-2 & 22,91 & 9,45 & \\
\hline & PC_recursos-3 & 9,2 & 9,98 & \\
\hline \multicolumn{5}{|c|}{ Efectos sobre la responsabilidad social: } \\
\hline \multirow[t]{4}{*}{ Alcance de mejoras } & RS_mejora-0 & 42,89 & $-1,78$ & \multirow{4}{*}{0,45} \\
\hline & RS_mejora-1 & 18,79 & 7,16 & \\
\hline & RS_mejora-2 & 21,62 & 8,94 & \\
\hline & RS_mejora-3 & 16,7 & 8,92 & \\
\hline \multirow[t]{4}{*}{ Cumplimiento normativo } & RS_norma-0 & 39,5 & $-2,20$ & \multirow{4}{*}{0,45} \\
\hline & RS_norma-1 & 16,47 & 6,78 & \\
\hline & RS_norma-2 & 21,81 & 8,16 & \\
\hline & RS_norma-3 & 22,22 & 8,86 & \\
\hline
\end{tabular}

* Posible valoración: (0) no efecto, o no relevante; (1) efecto reducido; (2) efecto intermedio; (3) efecto elevado. ** El \% de la inercia total capturada por el $1^{\circ}$ Factor del AC es del $74 \%$ (el $26 \%$ restante lo recoge el $2^{\circ}$ Factor). Fuente: Elaboración propia a partir del PITEC 2006.

\section{ANÁLISIS DESCRIPTIVO DEL DESEMPEÑO INNOVADOR PARA LAS EMPRESAS INNOVADORAS ESPAÑOLAS}

Una vez establecida nuestra medida del desempeño innovador para las empresas españolas con actividad innovadora, procedemos a caracterizar el nivel de desempeño alcanzado según el tipo de empresas. Para facilitar su interpretación, normalizamos la variable de forma que el valor 0 se corresponde con el desempeño innovador medio para el conjunto de empresas analizadas. En consecuencia, a la hora de realizar comparaciones entre empresas, un valor positivo (o negativo) indica haber alcanzado un nivel de desempeño superior (o inferior, respectivamente) a dicho valor de referencia medio. El conjunto de empresas 
analizadas es la muestra utilizada para la construcción del índice de desempeño innovador (9.432 empresas con actividades de innovación tecnológica del PITEC 2006). En la Tabla 6 se presenta el valor medio (desviación típica) para distintos tipos de empresas. Las variables de análisis seleccionadas hacen referencia a dos aspectos complementarios: características estructurales de las empresas (tipos de empresas según su tamaño, alcance geográfico, titularidad y sector de actividad $^{5}$ ) y políticas empresariales llevadas a cabo por la empresa en relación a la innovación tecnológica. Dentro de este último apartado se consideran siete variables: tipo de actividad de I+D interna según su carácter ocasional o continuo; personal dedicado a I+D interno; peso del gasto en I+D sobre la cifra de negocios; tipo de investigación llevada a cabo en relación al gasto corriente en I+D; posible cooperación con otras entidades en actividades de innovación; posible solicitud de patentes y modelos de utilidad.

En lo que respecta al valor medio, se pone de manifiesto que el perfil de las empresas con mayor nivel de desempeño innovador está asociado a empresas con un tamaño superior a 50 empleados, que operan en un ámbito de actuación internacional, de carácter privado con participación extranjera o centros de investigación y, como parece lógico, pertenece a sectores activos en innovación tecnológica. Por otra parte, las estrategias empresariales que más propician elevar el nivel del desempeño innovador en términos medios son: la realización de actividades de $\mathrm{I}+\mathrm{D}$ interna de forma continua, disponer de mayor personal dedicado específicamente a este tipo de actividades, incrementar la proporción del gasto en I+D en relación a las ventas, llevar a cabo investigación básica, cooperar con otras entidades y disponer de capacidad para solicitar patentes y modelos de utilidad. Si bien se trata de resultados predecibles, su constatación permite avalar la importancia de la medida del desempeño innovador construido. El valor añadido que proporciona este indicador es que integra razonablemente bien en una única variable la valoración de la empresa sobre un total de nueve efectos obtenidos de su actividad innovadora relativa al producto, proceso y responsabilidad social.

Estos resultados son coherentes con los obtenidos en otros estudios. De Propris (2000) y Chang (2003) concluyen que cuando las empresas cooperan en innovación con otras empresas o instituciones hay mayor probabilidad de obtener un elevado desempeño innovador que si no cooperan. Asimismo, De Propris (2000) también encuentra que la probabilidad de obtener un elevado desempeño innovador es mayor cuanto mayor es el porcentaje de gastos de I+D sobre las ventas. Y Díaz et al. (2006) observan una incidencia positiva del tamaño (número de empleados), de la pertenencia a sectores de elevada intensidad tecnológica y de la propiedad industrial (patentes y modelos de utilidad) en la probabilidad de innovar.

Sin embargo, De Propris (2000) concluye que la titularidad, el personal dedicado a actividades de I+D y el tamaño no parecen contribuir a explicar el desempeño innovador de las empresas. Díaz et al. (2006) encuentran que la presencia de propiedad extranjera en el capital influye de manera negativa en la probabilidad de innovar. Por su parte, Vaona y Pianta (2008) encuentran diferencias entre las empresas en cuanto a su desempeño innovador en función de su tamaño.

\footnotetext{
${ }^{5}$ Consideramos como sectores innovadores aquellos que son más activos en el ámbito de la innovación por contar con un alto porcentaje de empresas que realizan actividades de este tipo y una ratio de gastos de innovación frente a ventas elevado. Los sectores que llamamos innovadores son: Alimentación, bebidas y tabaco (CNAE 15 y 16), Química (CNAE 24), Maquinaria y transporte (CNAE del 29 al35) y Comunicaciones (CNAE 64).
} 
Tabla 6

Indicador del desempeño innovador de las empresas innovadoras de PITEC 2006

\begin{tabular}{lrccc}
\hline \multicolumn{2}{c}{ Tipo de empresas según características estructurales: } & $\%$ & Media & Desv. Típ. \\
\hline Número medio de empleados & & & & \\
& Entre 1 y 10 personas & 13.79 & -.1288011 & .454272 \\
Entre 11 y 50 personas & 39.09 & -.00135 & .42739 \\
Entre 51 y 100 personas & 14.76 & .0460729 & .4250199 \\
& Entre 101 y 200 personas & 10.75 & .0633428 & .4243895 \\
Mlcance geográfico & Más de 200 personas & 21.61 & .0216804 & .4447803 \\
& & & & \\
& Local & 9.20 & -.160695 & .447707 \\
& Nacional & 27.86 & -.0814725 & .4464479 \\
& Internacional & 62.93 & .0595675 & .4205203
\end{tabular}

Clase de empresa

$\begin{array}{rccc}\text { Pública } & 2.23 & -.0993542 & .418372 \\ \text { Privada nacional } & 86.06 & -.0034799 & .4381493 \\ \text { Privada con participación extranjera } & 10.17 & .0435968 & .4367192 \\ \text { Asociación o instituciones de investigación } & 1.55 & .0500093 & .4243703\end{array}$

Sector según el nivel de actividad en innovación tecnológica (IT)

Sectores menos activos en IT $\quad 65,82 \quad-.0481907 \quad 440982$

Sectores innovadores o más activos en IT (CNAE: 15, 16, $24,29-35,64)$

34,18 $\quad 0927939 \quad .4163179$

Tipo de empresas respecto a distintas políticas sobre I+D+i:

$\% \quad$ DI medio Std. Dev.

Tipo de actividad de I+D interna

Personal dedicado a I+D interno

$\begin{array}{rrrr}\text { No realiza } & 31.89 & -.1688403 & .4639952 \\ \text { De forma ocasional } & 14.55 & -.0227307 & .4375473 \\ \text { De forma continua } & 53.56 & .1067019 & .3863649\end{array}$

$\begin{array}{rccc}\text { Sin personal } & 31.89 & -.1688403 & .4639952 \\ \text { Entre 1 y 10 personas } & 51.69 & .0507375 & .4077688 \\ \text { Entre 11 y 50 personas } & 13.33 & .1609276 & .3660205 \\ \text { Entre 51 y 100 personas } & 1.81 & .2041619 & .3560771 \\ \text { Más de 100 personas } & 1.28 & .1927989 & .3843608\end{array}$

Porcentaje del gasto en I+D sobre cifra de negocios (distribución en cuartiles)

\begin{tabular}{llll} 
Partición cuartílica 1 & 25.00 & $-0,1814993$ & 0,465395 \\
Partición cuartílica 2 & 25.00 & 0,0552977 & 0,4219647 \\
Partición cuartílica 3 & 25.00 & 0,0835777 & 0,4005506 \\
Partición cuartílica 4 & 25.00 & 0,0426069 & 0,4089782 \\
\hline
\end{tabular}




\begin{tabular}{|c|c|c|c|}
\hline \multicolumn{4}{|l|}{ Tipo de investigación según gasto corriente en I+D } \\
\hline Ningún tipo de investigación & 31.89 & -.1688403 & .4639952 \\
\hline Aplicada y/o desarrollo tecnológico sin investigación básica & 61.93 & .0717814 & .4027699 \\
\hline Investigación básica & 6.18 & .1519668 & .3793625 \\
\hline \multicolumn{4}{|c|}{ Cooperación con otras entidades para actividades de innovación (entre 2004-06) } \\
\hline No coopera & 64.33 & -.0514148 & .4574196 \\
\hline Sí coopera & 35.67 & .0927423 & .3830508 \\
\hline \multicolumn{4}{|l|}{ Solicitud de Patentes (entre 2004-06) } \\
\hline No & 86.20 & -.0268225 & .4425713 \\
\hline Sí & 13.80 & .1674863 & .3649112 \\
\hline \multicolumn{4}{|l|}{ Modelos de utilidad (entre 2004-06) } \\
\hline No & 89.48 & -.0192592 & .4404965 \\
\hline Sí & 10.52 & .1638588 & .3766523 \\
\hline
\end{tabular}

En la línea de seguir ilustrando la sencillez de manejar un único indicador y con objeto de completar este análisis comparativo entre empresas, en los Gráficos 1 a 11 se muestra, a través de diagramas de caja, la correspondiente distribución de nuestra medida del desempeño innovador. De esta forma, además del valor máximo y mínimo alcanzado por el desempeño innovador en cada tipo de empresas, en la caja se indica el percentil 25 (aquel valor del desempeño innovador por debajo del cual se encuentra el $25 \%$ de las empresas con menor desempeño innovador); el percentil 50 o valor mediana, que se corresponde con el valor que delimita el $50 \%$ de las empresas con mayor y menor nivel de desempeño innovador y el percentil 75 que identifica el valor a partir del cual se encuentra el $25 \%$ de las empresas con mayor desempeño innovador.

Si bien este análisis gráfico corrobora los principales rasgos distintivos ya comentados para las empresas españolas según el nivel de desempeño innovador alcanzado, también permite extraer algún detalle adicional de carácter descriptivo. Así, por ejemplo, podemos observar que el tipo de empresas asociadas a menores niveles de desempeño innovador (empresas pequeñas, alcance local, titularidad pública, sectores menos activos en innovación, sin actividad en I+D interno, etc.) presentan una distribución del desempeño alcanzado más simétrica que el resto en torno a su valor mediana. Se cumple, además, que más del $50 \%$ de las empresas de este tipo presentan un desempeño innovador inferior al desempeño medio del conjunto completo de empresas, que por construcción toma valor 0 . Por su parte, la distribución del desempeño innovador de las empresas de mayor desempeño presenta una mayor concentración, o menor variabilidad, en torno a niveles más elevados del desempeño medio. Esta característica se aprecia especialmente en las empresas que operan en el ámbito internacional, en sectores de mayor actividad innovadora, con un desarrollo sistemático y personal especializado en la actividad de I+D de carácter interno, que cooperan con otras entidades y presentan capacidad para solicitar patentes y modelos de utilidad. Podría deducirse de esta observación, que este tipo de políticas estratégicas empresariales encaminadas a mejorar los resultados de la innovación conllevan, además de a un mayor desempeño innovador, a una mayor estabilidad (menor dispersión) en relación a los efectos positivos obtenidos. 
Este estudio exploratorio también nos permite deducir la no linealidad de los efectos esperados en relación a algunas variables. Así, por ejemplo, nuestro análisis pone de manifiesto que las empresas de mayor tamaño, y con mayor personal dedicado a actividades I+D interna, son las que presentan mayor desempeño innovador, ver Gráfico 1 y Gráfico 6, respectivamente. Esto puede deberse al logro de economías de escala y alcance que les permiten abordar un mayor número de proyectos de I+D y de mayor envergadura. Sin embargo, también captamos la no linealidad o proporcionalidad de esta asociación causa-efecto. A partir de un cierto tamaño comienzan a surgir deseconomías, posiblemente derivados de costes de coordinación y control, que pueden estabilizar o incluso incidir negativamente en el desempeño innovador.

\section{Gráfico 1}

\section{Desempeño innovador en función del tamaño de la empresa (número de empleados)}

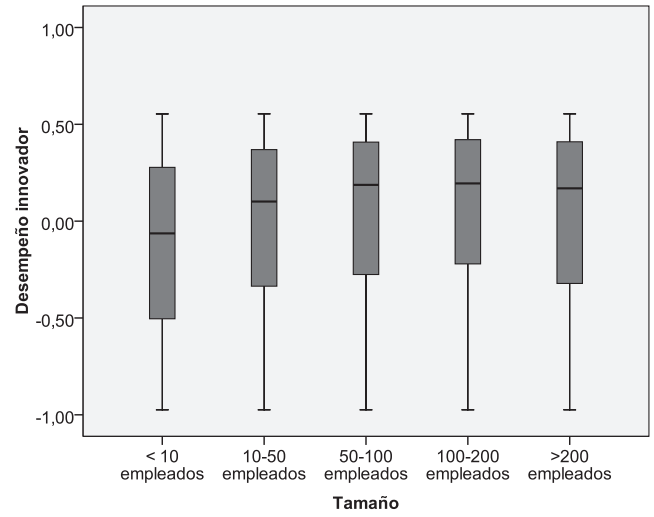

Gráfico 2

Desempeño innovador en función del alcance geográfico del mercado

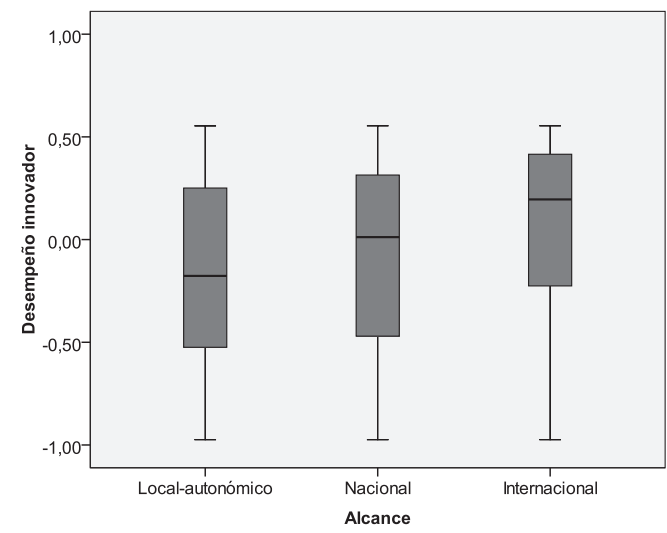




\section{Gráfico 3}

Desempeño innovador en función de la clase de empresa

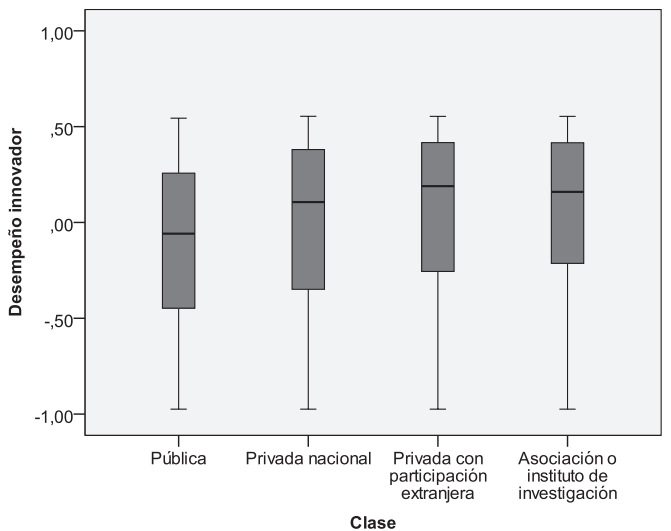

Gráfico 4

Desempeño innovador en función del sector de actividad

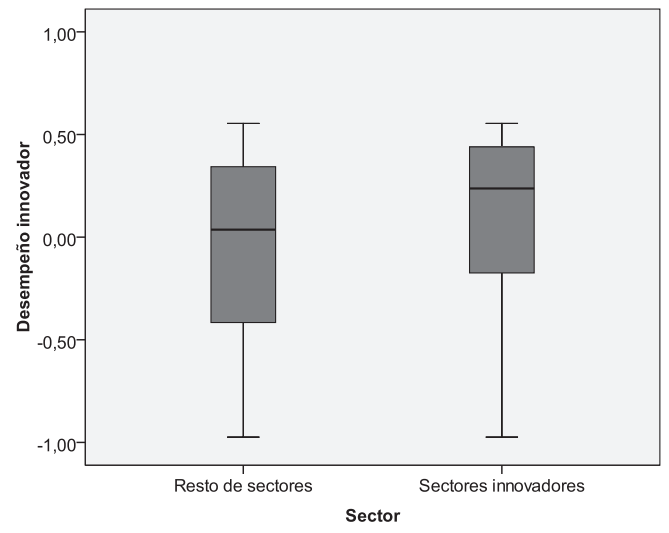




\section{Gráfico 5}

Desempeño innovador en función de si realiza actividades de I+D interna

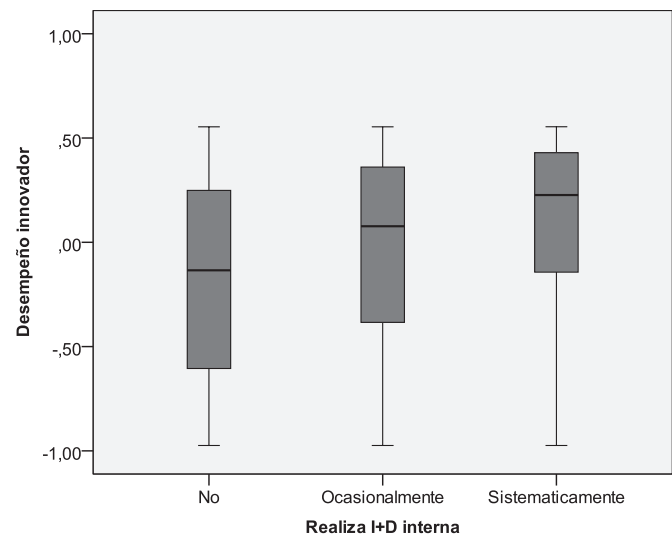

Gráfico 6

Desempeño innovador en función del número de empleados en actividades de I+D interna

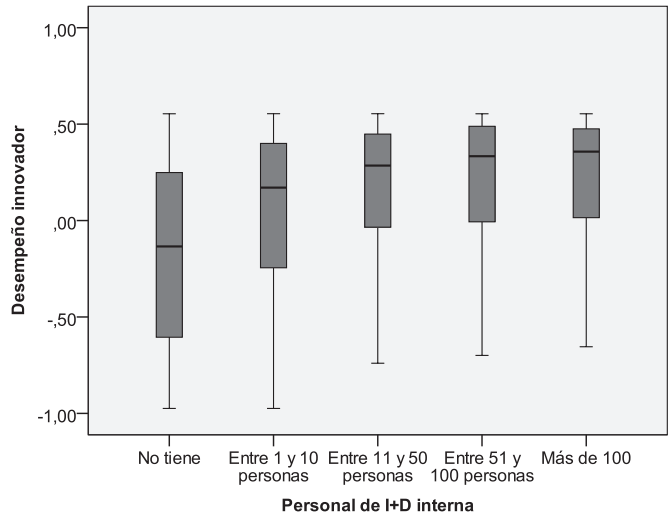




\section{Gráfico 7}

Desempeño innovador en función del gasto en innovación sobre la cifra de negocios

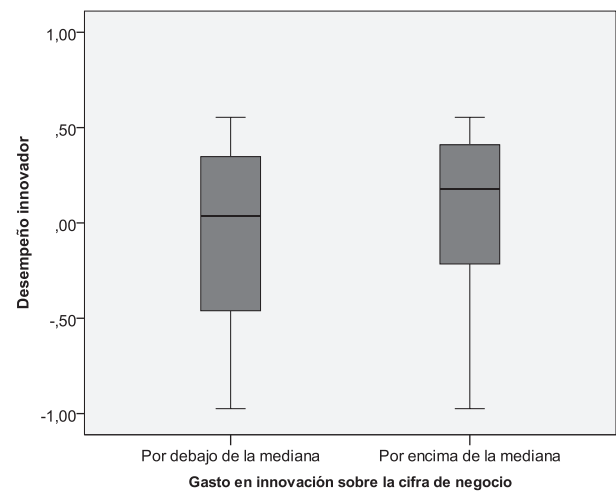

Gráfico 8

Desempeño innovador en función del tipo de investigación

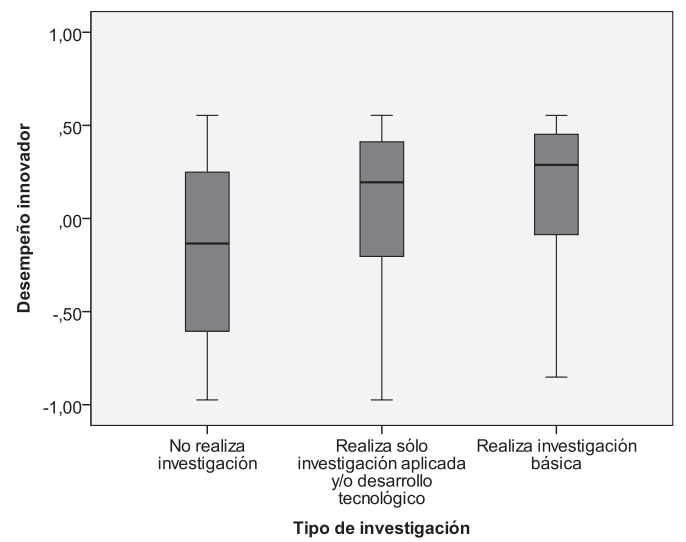




\section{Gráfico 9}

Desempeño innovador en función de si coopera en actividades de innovación

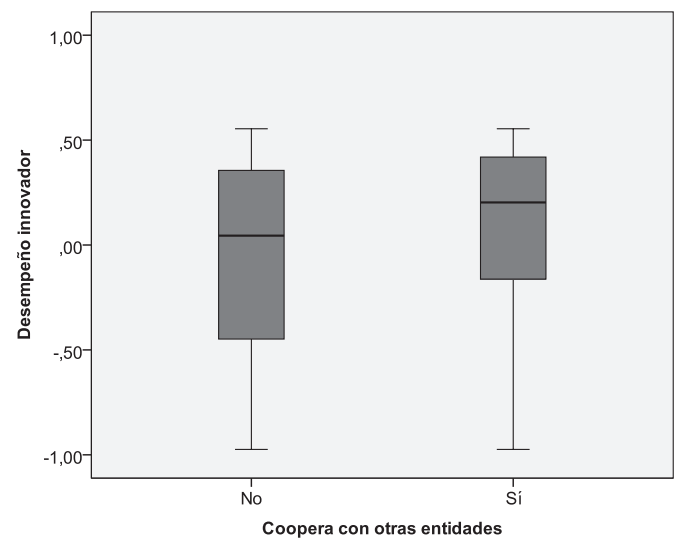

Gráfico 10

Desempeño innovador en función de si patenta

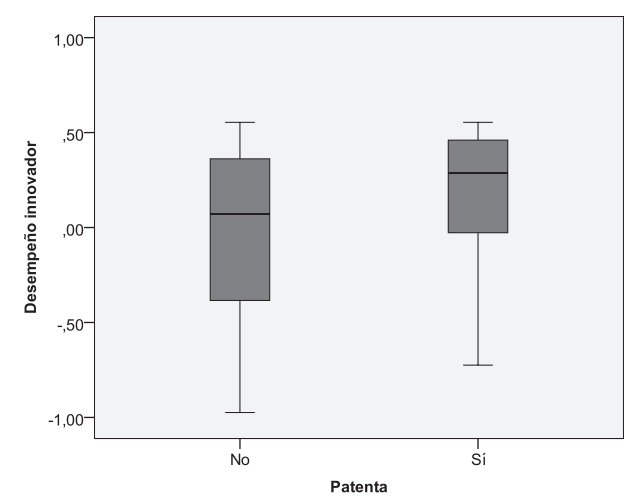




\section{Gráfico 11}

Desempeño innovador en función de si solicita modelos de utilidad

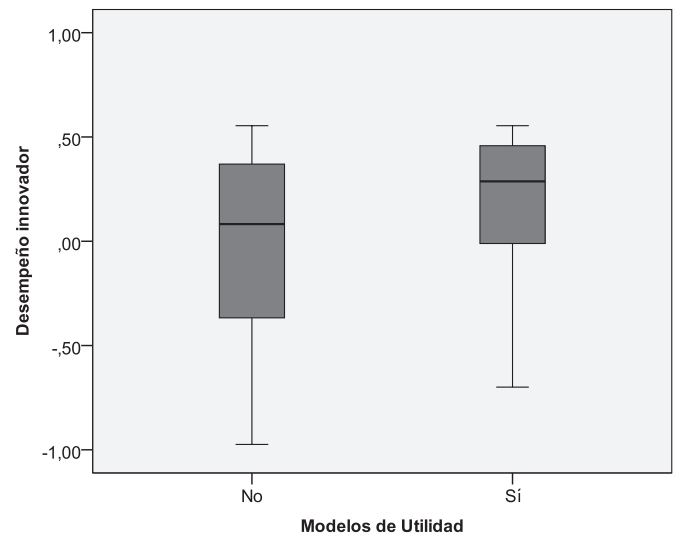

\section{CONCLUSIONES}

La principal aportación de este trabajo es la construcción de un único indicador para medir el desempeño innovador de las empresas españolas con actividad en innovación tecnológica. Para ello, se aplica la técnica estadística del análisis de correspondencias que permite integrar los efectos obtenidos de la actividad innovadora en tres dimensiones complementarias: efectos en los productos, en los procesos y en otros aspectos relativos a la sostenibilidad de la empresa.

El disponer de un indicador global del desempeño innovador es de suma importancia tanto para investigadores como para gestores. En el ámbito de la investigación académica, el desempeño innovador aparece como una variable intermedia entre ciertos procesos, políticas o estrategias empresariales y el desempeño de la empresa. Las actividades de innovación contribuyen a crear y mantener ventajas competitivas e involucran a distintas áreas empresariales (marketing, producción, finanzas y recursos humanos) y no se circunscriben al departamento de I+D (que puede darse el caso de que no exista). En el ámbito empresarial, los gestores necesitan de un indicador del desempeño de la innovación por dos razones básicas. En primer lugar, porque constituye un instrumento de gestión, al permitir detectar las oportunidades y necesidades existentes, ayudando al diseño de políticas y estrategias adecuadas para mejorar la competitividad de la empresa. Y en segundo lugar, este indicador también puede utilizarse como un instrumento de control, puesto que posibilita valorar la gestión de las actividades de I+D y los resultados de tales actividades, y así evaluar el logro de los objetivos fijados. Si las actividades de innovación permiten a la empresa alcanzar los resultados deseados, los gestores estarán dispuestos a asignar recursos a tales actividades. Esta información es de interés también para otros stakeholders de la empresa (accionistas, entidades financieras y administración pública).

Además, la existencia de un indicador del desempeño innovador de las empresas tiene implicaciones para los diseñadores de políticas públicas. En distintos ámbitos económicos 
se baraja el fomento de la innovación como una de las posibles soluciones a la actual crisis económica. El diseño de políticas públicas que potencien la innovación puede permitir un crecimiento económico sostenible. Por tanto, disponer de una medida del desempeño de las actividades de innovación de las empresas permite a los responsables públicos conocer cómo están evolucionando éstas en este área y tomar decisiones al respecto.

Este trabajo también incluye un estudio descriptivo del desempeño de las actividades de innovación tecnológica de las empresas innovadoras españolas, atendiendo a dos aspectos: características estructurales de las empresas (tamaño, alcance geográfico, titularidad y sector de actividad) y políticas empresariales en materia de I+D+i (carácter ocasional o continuo de la $\mathrm{I}+\mathrm{D}$ interna, número de empleados dedicados a $\mathrm{I}+\mathrm{D}$ interna, gasto en $\mathrm{I}+\mathrm{D}$ sobre la cifra de negocios, tipo de investigación, cooperación con otras entidades en actividades de innovación, y solicitud de patentes y modelos de utilidad).

Como resultado de este análisis se concluye que las empresas que presentan un mayor desempeño innovador tienen un número de empleados superior a 50, actúan en un ámbito internacional, son de carácter privado con participación extranjera o centros de investigación y pertenecen a sectores activos en innovación tecnológica. Asimismo, entre las políticas empresariales que más favorecen la obtención de un elevado desempeño innovador están: la continuidad en la realización de actividades de I+D interna, el aumento de la plantilla de personal dedicado a $\mathrm{I}+\mathrm{D}+\mathrm{i}$, el incremento de la proporción del gasto en $\mathrm{I}+\mathrm{D}$ en relación a las ventas, la realización de investigación básica, la cooperación en materia de I+D+i y la solicitud de patentes y modelos de utilidad.

No obstante, esta investigación presenta limitaciones. En primer lugar, el carácter descriptivo del análisis realizado no nos permite estimar con rigor estadístico los efectos de los distintos factores empresariales sobre el desempeño innovador, lo cual en sí mismo podría constituir el objetivo de una nueva línea de investigación. En efecto, el indicador construido en este artículo será empleado en un trabajo posterior sobre el impacto en el desempeño innovador de distintos factores determinantes relativos a características, políticas y estrategias de las empresas. En segundo lugar, al emplear el PITEC, estamos condicionados por los datos que éste nos ofrece. Así no podemos contar con una medida adecuada del impacto de las actividades de innovación tecnológica en los resultados económicos de la empresa, cuando en medidas anteriores del desempeño innovador es habitual la inclusión de indicadores de tipo financiero. El PITEC incluye como medida del impacto económico de las actividades de innovación tecnológica, el porcentaje de la cifra de negocios total que es debida a innovaciones en bienes y servicios introducidos en los tres años anteriores. Sin embargo, hemos optado por no incluir esta variable porque constituye una medida parcial del desempeño innovador de la empresa, al considerar únicamente el efecto de las innovaciones en producto, por tanto, la muestra se restringiría a las empresas que han llevado a cabo innovaciones de producto no considerándose a las empresas que sólo han realizado innovaciones de proceso. En compensación el uso del PITEC permite contar con una muestra muy amplia de empresas de diferentes sectores, lo que confiere más solidez a los resultados obtenidos en este trabajo.

A pesar de estas limitaciones, consideramos que el indicador desarrollado en este artículo constituye una medida adecuada del desempeño innovador de la empresa, por cuanto integra el impacto que las actividades de innovación tecnológica ha podido tener en los productos, en los procesos de producción, y en la sostenibilidad de la empresa. 


\section{REFERENCIAS BIBLIOGRÁFICAS}

AHUJA, G. y KATILA, R. (2001): “Technological Acquisitions and the Innovation Performance of Acquiring Firms: A Longitudinal Study”, Strategic Management Journal, Vol. 22, p. 197-220.

ALEGRE, J.; LAPIEDRA, R. y CHIVA, R. (2006): “A Measurement Scale for Product Innovation Performance", European Journal of Innovation Management, Vol. 9, №. 4, p. 333-346.

ATUAHENE-GIMA, K.; LI, H. y DE LUCA, L. M. (2006): “The Contingent Value of Marketing Strategy Innovativeness for Product Development Performance in Chinese New Technology Ventures", Industrial Marketing Management, Vol. 35, p. 359-372.

BIRCHALL, D. W. y TOVSTIGA, G. (2006): "Innovation Performance Measurement: Expert vs Practitioner Views", PICMET 2006 Proceedings, July, Istanbul, Turkey.

BLINDENBACH-DRIESSEN, F.; DALEN, J. y ENDE, J. (2010): "Subjective Performance Assessment of Innovation Projects", Journal of Product Innovation Management, Vol. 27, p. 572-592.

CHANG, Y. (2003): "Benefits of Co-operation on Innovative Performance: Evidence from Integrated Circuits and Biotechnology Firms in the UK and Taiwan", R\&D Management, Vol. 33, No. 4, p. 425-437.

CHEN, J.; CHEN, Y. y VANHAVERBEKE, W. (2011): "The Influence of Scope, Depth, and Orientation of External Tecnology Sources on the Innovative Performance of Chinese Firms", Technovation, Vol. 31, No. 8, p. 362-373.

CHEN, Y.; LIN, M. J. y CHANG, C. (2006): “The Influence of Intellectual Capital on New Product Development Performance -The Manufacturing Companies of Taiwan as an Example", Total Quality Management, Vol. 17, No. 10, p. 1323-1339.

DE BRENTANI, U. y KLEINSCHMIDT, E. J. (2004): "Corporate Culture and Commitment: Impact on Performance of International New Product Development Programs", Journal Production Innovation Management, Vol. 21, p. 309-333.

DE CAROLIS, D. M. (2003): "Competencies and Imitability in the Pharmaceutical Industry: An Analysis of Their Relationship with Firm Performance", Journal of Management, Vol. 29, Nº. 1, p. 27-50.

DE CAROLIS, D. M. y DEEDS, D. L. (1999): "The Impact of Stocks and Flows of Organizational Knowledge on Firm Performance: An Empirical Investigation of the Biotechnology Industry", Strategic Management Journal, Vol. 20, p. 953-968.

DE PROPRIS, L. (2000): "Innovation and Inter-firm Cooperation: The Case of the West Midlands", Economics of Innovation and New Technology, Vol. 9, p. 421-446.

DÍAZ DÍAZ, N.L; AGUIAR DÍAZ, I.; DE SAÁ PÉREZ P. (2006): "El Conocimiento Organizativo Tecnológico y la Capacidad de Innovación. Evidencia para la Empresa Industrial Española", Cuadernos de Economía y Dirección de la Empresa, №. 27, p. 33-59.

FRISHAMMAR, J. y HÖRTE, S. A. (2005): "Managing External Information in Manufacturing Firms: The Impact on Innovation Performance", Journal of Product Innovation Management, Vol. 22, p. 251-266.

FOSFURI, A. y TRIBÓ, J. A. (2008): "Exploring the Antecedents of Potential Absorptive Capacity and Its Impact on Innovation Performance", OMEGA, Vol. 36, p. 173-187.

GARCÍA, R. y CALANTONE R. (2002): "A critical look at technological innovation typology and innovativeness terminology: a literature review", Journal of Product Innovation Management, Vol 19, p. 110-132.

GREENACRE, M. J. (1991): "Interpreting Multiple Correspondence Analyses", Applied Stochastic Models and Data Analysis, Vol. 7, p. 195-210.

GRIFFIN, A. y PAGE, A. L. (1993): "An Interim Report on Measuring Product Development Success and Failure", Journal of Product Innovation Management, Vol. 10, p. 291-308. 
GRIFFIN, A. y PAGE, A. L. (1996): "PDMA Success Measurement Project: Recommended Measures for Product Development Success and Failures", Journal of Product Innovation Management, Vol. 13, p. 478-496.

GRUPP, H.; MOGGE, M. E. (2004): "Indicators for National Science and Technology Policy: How Robust are Composite Indicators?", Research Policy, Vol. 33, p. 1373-1384.

HAGEDOORN, J. y CLOODT, M. (2003): "Measuring Innovative Performance: Is There an Advantage in Using Multiple Indicators?", Research Policy, Vol. 32, p. 1365-1379.

HOOLEY, G.J.; GREENLEY, G.E.; CADOGAN, J.W. y FAHY, J. (2005): “The performance impact of marketing resources", Journal of Business Research, Vol. 58, pp. 18-27.

HUANG, X.; SOUTAR, G.N. y BROWN, A. (2004): "Measuring new product success: an empirical investigation of Australian SMEs", International Marketing Management, Vol. 33, p. 117-123.

JAFFE, A. B. y PALMER, K. (1997): "Environmental Regulation and Innovation: A Panel Study", Review of Economics and Statistics, Vol. 79, №. 4, p. 610-619.

KAMPIK, F. y DACHS, B. (2011): "The Innovative Performance of German Multinationals Abroad: Evidence from the European Community Innovation Survey", Industrial and Corporate Change, Vol. 20, $\mathrm{N}^{\circ}$. 2, p. 661-681.

KUSUNOKI, K.; NONAKA, I. y NAGATA, A. (1998): "Organizational Capabilities in Product Development of Japanese Firms: A Conceptual Framework and Empirical Findings", Organization Science, Vol. 9, $\mathrm{N}^{\circ}$. 6, p. 699-718.

LEE, Y. y O'CONNOR G.C. (2003): “The impact of communication strategy on launching new products: the moderating role of product innovativeness", Product Development \& Management Association, Vol. 20, p. 4-21.

LEONARD-BARTON, D. (1992): "Core Capabilities and Core Rigidities: A Paradox in Managing New Product Development", Strategic Management Journal, Vol. 13, p. 111-125.

LICHTENTHALER, U. (2009): "Absorptive Capacity, Environmental Turbulence, and the Complementarity of Organizational Learning Processes", Academy of Management Journal, Vol. 52, $\mathrm{N}^{\circ} .4$, p. 822-846.

MOLINA-CASTILLO, F.J. y MUNUERA-ALEMÁN, J.L. (2009a): "New Product Performance Indicators: Time Horizon and Importance Attributed by Managers", Technovation, Vol. 29, $\mathrm{N}^{\circ}$. 10, p. 714-724.

MOLINA-CASTILLO, F.J. y MUNUERA-ALEMÁN, J.L. (2009b): “The Joint Impact of Quality and Innovativeness on Short-Term New Product Performance", Industrial Marketing Management, Vol. 38, No. 8, p. 984-993.

MOLINA-MORALES, F.X.; MARTÍNEZ-FERNÁNDEZ, M.T. y TORLÒ, V.J. (2011): “The Dark Side of Trust: The Benefits, Costs and Optimal Levels of Trust for Innovation Performance", Long Range Planning, Vol. 44, No. 2, p. 118-133.

PAVELIN, S. y PORTER, A. (2008): "The Corporate Social Performance Content of innovation in the UK", Journal of Business Ethics, Vol. 80, p. 711-725.

PHILLIMORE, J. (2001): "Schumpeter, Schumacher and the Greening of Technology", Technology Analysis and Strategic Management, Vol.13, No. 1, p. 23-37.

PRAJOGO, D.I. y AHMED, P.K. (2006): "Relationships between Innovation Stimulus, Innovation Capacity, and Innovation Performance", $R \& D$ Management, Vol. 36, №. 5, p. 499-515.

SHER, P.J. y YANG, P.Y. (2005): "The Effects of Innovative Capabilities and R\&D Clustering on Firm Performance: The Evidence of Taiwan's Semiconductor Industry", Technovation, Vol. 25, p. 33-43.

SOUITARIS, V. (2002): "Firm-specific Competencies Determining Technological Innovation: A Survey in Greece", R\&D Management, Vol. 32, No. 1, p. 61-77.

UTTERBACK, J. y ABERNATHY, W. (1975): “A Dynamic Model of Product and Process Innovation", OMEGA, Vol. 3, №. 3, p. 639-656.

VAONA, A. y PIANTA, M. (2008): "Firm Size and Innovation in European Manufacturing”, Small Business Economics, Vol. 30, p. 283-299. 
